\title{
ON EXTREMAL LEAF STATUS AND INTERNAL STATUS
}

\author{
HAIYAN GUO ${ }^{1}$ AND Bo ZHOU ${ }^{2, *}$
}

\begin{abstract}
For a vertex $u$ of a tree $T$, the leaf (internal, respectively) status of $u$ is the sum of the distances from $u$ to all leaves (internal vertices, respectively) of $T$. The minimum (maximum, respectively) leaf status of a tree $T$ is the minimum (maximum, respectively) leaf statuses of all vertices of $T$. The minimum (maximum, respectively) internal status of a tree $T$ is the minimum (maximum, respectively) internal statuses of all vertices of $T$. We characterize those trees with the smallest (largest, respectively) extremal (minimum and maximum) leaf status and extremal (minimum and maximum) internal status, respectively. We also study the corresponding extremal problems for trees with given parameters, including diameter or maximum degree.
\end{abstract}

Mathematics Subject Classification. 05C12, 05C35.

Received July 19, 2021. Accepted January 17, 2022.

\section{INTRODUCTION}

Let $G$ be a connected graph of order $n \geq 2$ with vertex set $V(G)$. For $u, v \in V(G)$, the distance between $u$ and $v$ in $G$, denoted by $d_{G}(u, v)$, is the length of a shortest path connecting $u$ and $v$ in $G$. Let $\emptyset \neq A \subseteq V(G)$. For $u \in V(G)$, the $A$-status of $u$ in $G$ is defined as

$$
s_{G}(u, A)=\sum_{v \in A} d_{G}(u, v) .
$$

The minimum $A$-status of $G$ is $s_{A}(G)=\min \left\{s_{G}(u, A): u \in V(G)\right\}$, while the maximum $A$-status of $G$ is $S_{A}(G)=\max \left\{s_{G}(u, A): u \in V(G)\right\}$. The $A$-centroid (or $A$-median) of $G$ is defined as $\left\{u \in V(G): s_{G}(u, A)=\right.$ $\left.s_{A}(G)\right\}$.

Let $s_{G}(u)=s_{G}(u, V(G))$ for $u \in V(G), s(G)=s_{V(G)}(G)$ and $S(G)=S_{V(G)}(G)$. Then $s_{G}(u)$ is the status (or transmission) of $u$ in $G[4,6,19], s(G)$ is the minimum status of $G$, and $S(G)$ is the maximum status of $G$. Both minimum and maximum statuses have been studied extensively, and it should be noted that the minimum (maximum, respectively) status appeared also in its normalized form divided by $n-1$ that is called the proximity (remoteness, respectively) of the graph, see, e.g., [1-3,5,7-9,11,12,15,16,21]. Some related works may be found in $[10,13,18,20]$.

Let $T$ be a tree. For $u \in V(T)$, denote by $N_{T}(u)$ the set of vertices adjacent to $u$ in $T$ and the cardinality of $N_{T}(u)$ is the degree of $u$ in $T$, denoted by $\delta_{T}(u)$. A vertex of degree one in a tree is called a leaf and a vertex

Keywords. Minimum leaf status, maximum leaf status, minimum internal status, maximum internal status, extremal problem.

1 School of Computer Science, South China Normal University, Guangzhou 510631, P.R. China.

2 School of Mathematical Sciences, South China Normal University, Guangzhou 510631, P.R. China.

*Corresponding author: zhoubo@scnu.edu.cn

(c) The authors. Published by EDP Sciences, ROADEF, SMAI 2022 
of degree at least two in a tree is called an internal vertex. Let $L(T)$ and $I(T)$ be the set of leaves and the set of internal vertices of $T$, respectively.

Slater [17] studied the structure of the $A$-centroid of a tree $T$ with $\emptyset \neq A \subseteq V(T)$. For example, it was shown in Theorem 5 of [17] that the $A$-centroid induces a path in a tree for any subset $A$. Another related concept is called $A$-center, which is defined to be the set $\left\{u \in V(T): e_{A}(u, T)=\min \left\{e_{A}(w, T): w \in V(T)\right\}\right\}$, where $e_{A}(u, T)=\max \left\{d_{T}(u, v): v \in A\right\}$. It was shown in [17] that $L(T)$-center and $L(T)$-centroid have quite different properties.

The minimum leaf status (internal status, respectively) of $T$ is defined to be the minimum $L(T)$-status $(I(T)$-status, respectively), denoted by $l s(T)(i s(T)$, respectively). That is,

$$
l s(T)=s_{L(T)}(T) \text { and } i s(T)=s_{I(T)}(T) .
$$

The maximum leaf status (internal status, respectively) of $T$ is defined to be the maximum $L(T)$-status $(I(T)$ status, respectively), denoted by $L S(T)(I S(T)$, respectively). That is,

$$
L S(T)=S_{L(T)}(T) \text { and } I S(T)=S_{I(T)}(T) .
$$

In this paper, we study the extremal (minimum, maximum) leaf status (internal status, respectively). The structures of the trees with maximum and minimum extremal (minimum, maximum) leaf status (internal status, respectively) are identified. A somewhat related but different extremal problem studied is the characterization of the trees with maximum distance between the $L(T)$-centroid and the centroid or the $I(T)$-centroid, and maximum distance between the $I(T)$-centroid and the centroid, respectively, where, for two subsets $A$ and $B$ of vertices of a connected graph $G$, the distance between $A$ and $B$ is the smallest distance between a vertex from $A$ and a vertex from $B$ in $G$, see [20].

In the context of communication networks, the status of a vertex is viewed as the cost of the vertex to the whole network, which measures the closeness centrality of the vertex in the network. The leaf status (internal status, respectively) of a vertex may be viewed as the cost of the vertex to the leaves (internal vertices, respectively) of a network of tree structure. The minimum leaf status (internal status, respectively) models locating a central facility in a network involving minimizing the sum of its distances from the leaf (internal, respectively) sources of flow to it, where the distance may be appropriately weighted to reflect the associated flow volume and/or cost.

\section{Preliminaries}

The diameter of a connected graph $G$ is the maximum distance between two vertices. Denote by $S_{n}$ and $P_{n}$ the star and the path of order $n$, respectively. A double star is a tree with diameter 3 , which is obtainable by adding an edge between the centers of two nontrivial stars.

For a vertex $u$ of a nontrivial tree $T$, the components of $T-u$ are called the branches of $T$ at $u$. For $A \subseteq V(T)$, the $A$-branch-weight of $u$ in $T$, denoted by $b w_{T}(u, A)$, is defined to be

$$
\max \{|A \cap V(B)|: B \text { is a branch of } T \text { at } u\} .
$$

For a tree $T$, a vertex in the $A$-centroid is called an $A$-centroid vertex. The following lemma is a restatement of Theorem 8 from [17].

Lemma 2.1. Let $T$ be a tree of order $n \geq 2$. Then $u$ is an $A$-centroid vertex if and only if $b w_{T}(u, A) \leq b w_{T}(v, A)$ for any $v \in V(T)$.

For $u, v \in V(T)$, denote by $n_{T}(u, v \mid A)$ the number of vertices in $A$ closer to $u$ than to $v$. Let $T$ be a tree with $u \in V(T)$. For $A=L(T), I(T)$, Wang ([20], Prop. 3.1) stated that $u$ is an $A$-centroid vertex of $T$ if and only if $n_{T}(u, v \mid A) \geq n_{T}(v, u \mid A)$ for any $v \in N_{T}(u)$.

We give a somewhat easier necessary and sufficient condition for a vertex of a tree $T$ to be an $A$-centroid vertex for $A=L(T), I(T)$. 
Lemma 2.2. Let $T$ be a tree of order $n \geq 3$ with $u \in V(T)$. For $A=L(T), I(T), u$ is an $A$-centroid vertex of $T$ if and only if $b w_{T}(u, A) \leq \frac{|A|}{2}$.

Proof. Let $r=\delta_{T}(u)$ and $N_{T}(u)=\left\{u_{1}, \ldots, u_{r}\right\}$. For $i=1, \ldots, r$, let $B_{i}$ be the branch of $T$ at $u$ containing $u_{i}$ and let $a_{i}=\left|A \cap V\left(B_{i}\right)\right|$. Assume that $a_{1} \geq \cdots \geq a_{r}$. Then, by definition, $b w_{T}(u, A)=a_{1}$.

Suppose that $b w_{T}(u, A) \leq \frac{|A|}{2}$, i.e., $a_{1} \leq \frac{|A|}{2}$. For any $v \in V(T) \backslash\left(V\left(B_{1}\right) \cup\{u\}\right)$, say $v \in V\left(B_{i}\right)$ with $2 \leq i \leq r$, as $T-V\left(B_{i}\right)$ is a subtree of a branch at $v$, we have $b w_{T}(v, A) \geq\left|A \cap\left(V(T) \backslash V\left(B_{i}\right)\right)\right| \geq \sum_{j=1}^{r} a_{j}-a_{i} \geq a_{1}=$ $b w_{T}(u, A)$. If $A=L(T)$, then $\sum_{j=1}^{r} a_{j}=|A|$, so $\sum_{j=2}^{r} a_{j} \geq \frac{|A|}{2}$, and for any $v \in V\left(B_{1}\right)$, we have $b w_{T}(v, A) \geq$ $\left|A \cap\left(V(T) \backslash V\left(B_{1}\right)\right)\right| \geq \sum_{j=2}^{r} a_{j} \geq \frac{|A|}{2} \geq b w_{T}(u, A)$. If $A=I(T)$, then $\sum_{j=1}^{r} a_{j}=|A|-1$, so $\sum_{j=2}^{r} a_{j} \geq \frac{|A|}{2}-1$, and for any $v \in V\left(B_{1}\right)$, we have $b w_{T}(v, A) \geq\left|A \cap\left(V(T) \backslash V\left(B_{1}\right)\right)\right| \geq 1+\sum_{j=2}^{r} a_{j} \geq \frac{|A|}{2} \geq b w_{T}(u, A)$. Therefore $b w_{T}(u, A) \leq b w_{T}(v, A)$ for any $v \in V(T)$, which implies that $u$ is an $A$-centroid vertex of $T$ by Lemma 2.1.

Conversely, suppose that $u$ is an $A$-centroid vertex of $T$.

Case 1. $A=L(T)$.

If $|A|=2$, then $T \cong P_{n}$ and $u$ may be any vertex. Then $b w_{T}(u, A)=1 \leq \frac{|A|}{2}$. Suppose that $|A| \geq 3$. If $b w_{T}(u, A)>\frac{|A|}{2}$, i.e., $a_{1}>\frac{|A|}{2}$, then $\sum_{i=2}^{r} a_{i}<\frac{|A|}{2}$, so $s_{T}(u, L(T))-s_{T}\left(u_{1}, L(T)\right)=a_{1}-\sum_{i=2}^{r} a_{i}>0$, implying that $s_{T}(u, L(T))>s_{T}\left(u_{1}, L(T)\right)$, a contradiction. It follows that $b w_{T}(u, A) \leq \frac{|A|}{2}$.

Case 2. $A=I(T)$.

If $|A|=1$, then $T \cong S_{n}$ and $u$ is the center. If $|A|=2$, then $T$ is a double star and $u$ may be either of the internal vertices. So we have $b w_{T}(u, A)=0,1 \leq \frac{|A|}{2}$ if $|A|=1,2$. Suppose that $|A| \geq 3$. If $b w_{T}(u, A)>\frac{|A|}{2}$, i.e., $a_{1}>\frac{|A|}{2}$, then $\sum_{i=2}^{r} a_{i}<\frac{|A|}{2}-1$, so $s_{T}(u, I(T))-s_{T}\left(u_{1}, I(T)\right)=a_{1}-1-\sum_{i=2}^{r} a_{i}>0$, a contradiction. It follows that $b w_{T}(u, A) \leq \frac{|A|}{2}$.

By combining Cases 1 and 2 , we have $b w_{T}(u, A) \leq \frac{|A|}{2}$.

A leaf peripherian vertex of a tree $T$ on $n$ vertices is a vertex of $T$ with maximum leaf status. Note that every vertex of $P_{n}$ is a leaf peripherian vertex and $L S\left(P_{n}\right)=n-1$. In the following lemma, we show that a leaf peripherian vertex of a tree that is not a path must be a leaf.

Lemma 2.3. Let $T$ be a tree that is not a path. Let $u \in V(T)$. If $u$ is a leaf peripherian vertex of $T$, then $u \in L(T)$.

Proof. We prove the lemma by contradiction. Suppose that $u$ is a leaf peripherian vertex of $T$ but $u \notin L(T)$. Then $\delta_{T}(u) \geq 2$. Let $r=\delta_{T}(u)$ and $N_{T}(u)=\left\{u_{1}, \ldots, u_{r}\right\}$, where $r \geq 2$. For $i=1, \ldots, r$, let $B_{i}$ be the branch of $T$ at $u$ containing $u_{i}, L_{i}=L(T) \cap V\left(B_{i}\right)$ and $a_{i}=\left|L_{i}\right|$. Assume that $a_{1} \leq \cdots \leq a_{r}$.

Suppose first that $r=2$ and $a_{2}=a_{1}$. As $T$ is not a path, we have $a_{2}=a_{1} \geq 2$. Let $v \in L_{1}$ and let $z$ be the unique vertex adjacent to $v$ in $T$. Then

$$
\begin{aligned}
& s_{T}(u, L(T))=\sum_{w \in L_{1} \backslash\{v\}} d_{T}(u, w)+d_{T}(u, v)+\sum_{w \in L_{2}} d_{T}(u, w), \\
& s_{T}(v, L(T))=\sum_{w \in L_{1} \backslash\{v\}} d_{T}(v, w)+\sum_{w \in L_{2}}\left(d_{T}(v, u)+d_{T}(u, w)\right),
\end{aligned}
$$

and so

$$
\begin{aligned}
s_{T}(v, L(T))-s_{T}(u, L(T)) & =\sum_{w \in L_{1} \backslash\{v\}}\left(d_{T}(v, w)-d_{T}(u, w)\right)-d_{T}(u, v)+\sum_{w \in L_{2}} d_{T}(v, u) \\
& >\sum_{w \in L_{1} \backslash\{v\}}\left(d_{T}(z, w)-d_{T}(u, w)\right)-d_{T}(u, v)+\sum_{w \in L_{2}} d_{T}(v, u)
\end{aligned}
$$




$$
\begin{aligned}
& \geq-\sum_{w \in L_{1} \backslash\{v\}} d_{T}(z, u)-d_{T}(u, v)+\sum_{w \in L_{2}} d_{T}(v, u) \\
& =-\sum_{w \in L_{1} \backslash\{v\}}\left(d_{T}(v, u)-1\right)-d_{T}(u, v)+\sum_{w \in L_{2}} d_{T}(v, u) \\
& =\sum_{w \in L_{1} \backslash\{v\}} 1 \\
& =a_{1}-1 \\
& >0 .
\end{aligned}
$$

Thus $s_{T}(v, L(T))>s_{T}(u, L(T))$. This implies that $u$ cannot be a leaf peripherian vertex of $T$, a contradiction.

Suppose next that $r=2$ and $a_{2}>a_{1}$, or $r \geq 3$. Then

$$
\begin{aligned}
s_{T}(u, L(T)) & =\sum_{v \in L_{1}} d_{T}(u, v)+\sum_{j=2}^{r} \sum_{v \in L_{j}} d_{T}(u, v), \\
s_{T}\left(u_{1}, L(T)\right) & =\sum_{v \in L_{1}}\left(d_{T}(u, v)-1\right)+\sum_{j=2}^{r} \sum_{v \in L_{j}}\left(d_{T}(u, v)+1\right),
\end{aligned}
$$

and so

$$
\begin{aligned}
s_{T}\left(u_{1}, L(T)\right)-s_{T}(u, L(T)) & =\sum_{v \in L_{1}}(-1)+\sum_{j=2}^{r} \sum_{v \in L_{j}} 1 \\
& =\sum_{i=2}^{r} a_{i}-a_{1} \\
& >0 .
\end{aligned}
$$

Thus $s_{T}\left(u_{1}, L(T)\right)>s_{T}(u, L(T))$. This implies that $u$ is not a leaf peripherian vertex of $T$, also a contradiction.

Therefore, $u \in L(T)$, as desired.

An internal peripherian vertex of a tree $T$ is a vertex of $T$ with maximum internal status.

Lemma 2.4. Let $T$ be a tree. Suppose that $u$ is an internal peripherian vertex of $T$. Then $u \in L(T)$.

Proof. We prove the lemma by contradiction. Suppose that $u \notin L(T)$. Then $\delta_{T}(u) \geq 2$.

If there is a vertex $v \in N_{T}(u)$ with $\delta_{T}(v)=1$, then it is obvious that $s_{T}(v, I(T))>s_{T}(u, I(T))$, a contradiction. So $\delta_{T}(v) \geq 2$ for any $v \in N_{T}(u)$. Let $r=\delta_{T}(u)$ and $N_{T}(u)=\left\{u_{1}, \ldots, u_{r}\right\}$, where $r \geq 2$. For $i=1, \ldots, r$, let $B_{i}$ be the branch of $T$ at $u$ containing $u_{i}, I_{i}=I(T) \cap V\left(B_{i}\right)$ and $a_{i}=\left|I_{i}\right|$. Assume that $a_{1} \leq \cdots \leq a_{r}$. Let $z \in I_{1}$. Then

$$
\begin{aligned}
& s_{T}(u, I(T))=\sum_{w \in I_{1} \backslash\{z\}} d_{T}(u, w)+d_{T}(u, z)+\sum_{i=2}^{r} \sum_{w \in I_{i}} d_{T}(u, w), \\
& s_{T}(z, I(T))=\sum_{w \in I_{1} \backslash\{z\}} d_{T}(z, w)+d_{T}(z, u)+\sum_{i=2}^{r} \sum_{w \in I_{i}}\left(d_{T}(z, u)+d_{T}(u, w)\right),
\end{aligned}
$$

and so

$$
s_{T}(z, I(T))-s_{T}(u, I(T))=\sum_{w \in I_{1} \backslash\{z\}}\left(d_{T}(z, w)-d_{T}(u, w)\right)+\sum_{i=2}^{r} \sum_{w \in I_{i}} d_{T}(z, u)
$$




$$
\begin{aligned}
& \geq-d_{T}(z, u)\left(a_{1}-1\right)+d_{T}(z, u) \sum_{i=2}^{r} a_{i} \\
& =d_{T}(z, u)\left(-a_{1}+1+\sum_{i=2}^{r} a_{i}\right) \\
& >0 .
\end{aligned}
$$

Thus $s_{T}(z, I(T))>s_{T}(u, I(T))$, a contradiction.

A tree is starlike if it has at most one vertex of degree greater than 2. So, a star and a path are both particular starlike trees.

A diametric path of a tree is a longest path in this tree (whose length equals the diameter). Evidently, the terminal vertices of a diametric path of any nontrivial tree are leaves.

A caterpillar is a tree such that the deletion of all leaves outside a diametric path (if any exists) yields a path.

An edge $u v$ in a tree $T$ is a leaf edge of $T$ at $u$ if $v$ is a leaf and $u$ is an internal vertex of $T$.

For a tree $T$ with $u w \in E(T)$ and $v w \notin E(T)$, if $T^{\prime}=T-u w+v w$ is a tree, then we also say that $T^{\prime}$ is obtained from $T$ by moving the edge $u w$ from $u$ to $v$.

A hanging path at a vertex $u$ of a tree $T$ is a path $u u_{1} \ldots u_{\ell}$ with $\delta_{T}(u) \geq 3, \delta_{T}\left(u_{\ell}\right)=1$ and if $\ell \geq 2$, $\delta_{T}\left(u_{i}\right)=2$ for $i=1, \ldots, \ell-1$.

Let $P$ be a path in a tree $T$. For $v \in V(T) \backslash V(P)$, the distance between $v$ and $P$ is defined to be $d_{T}(v, P)=$ $\min \left\{d_{T}(v, w): w \in V(P)\right\}$.

\section{Minimum Leaf status}

Theorem 3.1. Let $T$ be a tree of order $n \geq 2$. Then

$$
l s(T) \geq n-1
$$

with equality if and only if $T$ is starlike.

Proof. Let $u$ be an $L(T)$-centroid vertex. Every vertex other than $u$ lies on some path from $u$ to a leaf (including the leaves themselves), so $s_{T}(u, L(T)) \geq n-1$ with equality if and only if every edge of $T$ lies on exactly one path from $u$ to a leaf, equivalently, $T$ is starlike.

For integers $n, a$ and $b$ with $1 \leq a, b \leq \frac{n-2}{2}$, let $T_{n ; a, b}$ be the tree of order $n$ obtained from two stars $S_{a+1}$ and $S_{b+1}$ by connecting their centers by a path of length $n-a-b-1$. For convenience, let $T_{n, a}=T_{n ; a, a}$.

Theorem 3.2. Let $T$ be a tree of order $n$ with diameter $d$, where $3 \leq d \leq n-1$. Then

$$
l s(T) \leq \begin{cases}\frac{(n-d+1) d}{2} & \text { if } n-d \text { is odd } \\ \frac{(n-d) d}{2}+1 & \text { if } n-d \text { is even. }\end{cases}
$$

If $n-d$ is odd, then equality holds if and only if $T \cong T_{n,(n-d+1) / 2}$. If $n-d$ is even, then equality holds if and only if $T \cong T_{n ;(n-d) / 2,(n-d+2) / 2}$, or $d \geq 4$ and $T$ is isomorphic to a tree obtained from $T_{n-1,(n-d) / 2}$ by adding a leaf edge at a vertex of degree two.

Proof. If $d=n-1$, then the result is trivial as $n-d=1$ is odd, $T \cong P_{n}$ and $l s(T)=n-1=\frac{(n-d+1) d}{2}$.

Suppose that $d \leq n-2$. Let $T$ be a tree of order $n$ with diameter $d$ that maximizes the minimum leaf status. Let $x$ be an $L(T)$-centroid vertex. Let $r=\delta_{T}(x)$ and $N_{T}(x)=\left\{y_{1}, \ldots, y_{r}\right\}$. For $i=1, \ldots, r$, let $B_{i}$ be the branch of $T$ at $x$ containing $y_{i}$ and let $a_{i}=\left|L(T) \cap V\left(B_{i}\right)\right|$. 
Suppose that $r=2$. By Lemma 2.2, $a_{1}, a_{2} \leq \frac{|L(T)|}{2}$. So $a_{1}=a_{2}=\frac{|L(T)|}{2} \geq 2$. Choose a vertex $z \in V\left(B_{1}\right)$ with $\delta_{T}(z) \geq 3$ such that $d_{T}(x, z)$ is as small as possible. Then $b w_{T}(z, L(T))=a_{2}=\frac{|L(T)|}{2}$. So $z$ is an $L(T)$-centroid vertex by Lemma 2.2. Therefore, we may assume that $r \geq 3$. Let $P:=x_{0} x_{1} \ldots x_{d}$ be an arbitrary diametric path of $T$.

Suppose that $x$ lies outside any diametric path. By Lemma 2.2, $x$ is not a leaf. So $d_{T}(x, P)=d_{T}\left(x, x_{i}\right)$ for some $i$ with $3 \leq i \leq d-3$. Assume that $x_{i} \in V\left(B_{r}\right)$. Let $\ell$ be the length of a longest path from a leaf of $T$ in $\cup_{j=1}^{r-1} V\left(B_{j}\right)$ to $x_{i}$. Then $\ell \leq \min \{i-1, d-i-1\}$.

Claim 1. $a_{j} \leq a_{r}$ for each $j=1, \ldots, r-1$.

Suppose that this is not true. Then $a_{j}>a_{r}$ for some $j$ with $1 \leq j \leq r-1$. Let $T^{\prime}$ be the tree obtained from $T$ by moving the edges $x y_{k}$ with $1 \leq k \leq r-1$ and $k \neq j$ from $x$ to $y_{r}$. Note that the diameter of $T^{\prime}$ is $d$ and $L\left(T^{\prime}\right)=L(T)$. As $a_{j}>a_{r}$, we have $b w_{T^{\prime}}\left(y_{r}\right)=\max \left\{a_{1}, \ldots, a_{r-1}\right\}=b w_{T}(x)$. By Lemma $2.2, y_{r}$ is an $L\left(T^{\prime}\right)$-centroid vertex. Denote by $L_{k}$ the set of leaves of $T$ in $B_{k}$ for $k=j, r$. Then

$$
\begin{aligned}
l s\left(T^{\prime}\right)-l s(T) & =s_{T^{\prime}}\left(y_{r}, L\left(T^{\prime}\right)\right)-s_{T}(x, L(T)) \\
& =\sum_{z \in L_{j}} 1+\sum_{z \in L_{r}}(-1) \\
& =a_{j}-a_{r}>0,
\end{aligned}
$$

so $l s\left(T^{\prime}\right)>l s(T)$, a contradiction. This proves Claim 1 .

Let $w$ be the vertex on the path from $x$ to $x_{i}$ with degree at least three such that $d_{T}(w, x)$ is as small as possible.

Claim 2. $a_{1}=\cdots=a_{r}$.

Suppose that this is not true. By Claim $1, a_{j} \leq a_{r}$ for each $j=1, \ldots, r-1$, so we have $a_{j_{0}}<a_{r}$ for some $j_{0}=1, \ldots, r-1$. Let $T^{*}$ be the tree obtained from $T$ by moving the edges $x y_{k}$ with $1 \leq k \leq r-1$ and $k \neq j_{0}$ from $x$ to $y_{j_{0}}$. As $\ell \leq \min \{i-1, d-i-1\}$, the diameter of $T^{*}$ is $d$.

Case i. $\delta_{T}\left(y_{j_{0}}\right) \geq 2$.

Let $T_{0}$ be the maximal subtree of $T-x y_{j_{0}}$ containing $y_{j_{0}}$. Note that the branches of $T^{*}-y_{j_{0}}$ are $B_{k}$ with $1 \leq k \leq r-1$ and $k \neq j_{0}, T\left[\{x\} \cup V\left(B_{r}\right)\right]$, and the branches of $T_{0}$ at $y_{j_{0}}$. As $a_{k} \leq a_{r}$ for each $k=1, \ldots, r-1$, we have by Lemma 2.2 that $b w_{T^{*}}\left(y_{j_{0}}\right)=\max \left\{a_{k}: k=1, \ldots, r, k \neq j_{0}\right\}=a_{r}=b w_{T}(x) \leq \frac{|L(T)|}{2}=\frac{\left|L\left(T^{*}\right)\right|}{2}$ and so $y_{j_{0}}$ is an $L\left(T^{*}\right)$-centroid vertex. Thus $l s(T)=s_{T}(x, L(T))<s_{T}(x, L(T))+a_{r}-a_{j_{0}}=s_{T^{*}}\left(y_{j_{0}}, L\left(T^{*}\right)\right)=$ $l s\left(T^{*}\right)$, a contradiction.

Case ii. $\delta_{T}\left(y_{j_{0}}\right)=1$.

Suppose that $a_{r}=\frac{|L(T)|}{2}$. Then $w=x_{i}$, otherwise denote by $\beta_{k}$ the number of leaves of $T$ in branches at $w$ not containing its neighbors in the path from $x$ to $x_{i}$, where $k=1, \ldots, \delta_{T}(w)-2$. Then $b w_{T}(w)=$ $\max \left\{a_{1}+\cdots+a_{r-1}, \beta_{1}, \ldots, \beta_{\delta_{T}(w)-2}, a_{r}-\left(\beta_{1}+\cdots+\beta_{\delta_{T}(w)-2}\right)\right\}=a_{1}+\cdots+a_{r-1}=\frac{|L(T)|}{2}$, and $w$ is an $L(T)$-centroid vertex by Lemma 2.2. Let $w^{\prime}$ be the neighbor of $w$ in the branch of $T$ at $w$ containing $x_{i}$. Let $T^{\prime}$ be the tree obtained from $T$ by moving the edges incident to $w$ outside the path connecting $x$ and $x_{i}$ from $w$ to $w^{\prime}$. Then $b w_{T^{\prime}}\left(w^{\prime}\right)=\frac{|L(T)|}{2}=\frac{\left|L\left(T^{\prime}\right)\right|}{2}$. Thus $w^{\prime}$ is an $L\left(T^{\prime}\right)$-centroid vertex by Lemma 2.2. It is easy to see that $s_{T^{\prime}}\left(w^{\prime}, L\left(T^{\prime}\right)\right)-s_{T}(w, L(T))=a_{1}+\cdots+a_{r-1}-\left(a_{r}-\left(\beta_{1}+\cdots+\beta_{\delta_{T}(w)-2}\right)\right)=\beta_{1}+\cdots+\beta_{\delta_{T}(w)-2}>0$, implying that $l s\left(T^{\prime}\right)>l s(T)$, a contradiction. It thus follows that $w=x_{i}$. Let $T^{\prime \prime}=T-x y_{j_{0}}+x_{1} y_{j_{0}}$. As $b w_{T^{\prime \prime}}\left(x_{i}\right) \leq a_{r}=\frac{|L(T)|}{2}=\frac{\left|L\left(T^{\prime \prime}\right)\right|}{2}, x_{i}$ is an $L\left(T^{\prime \prime}\right)$-centroid vertex by Lemma 2.2. As $d_{T}\left(x, x_{i}\right)<i-1$, we have $l s\left(T^{\prime \prime}\right)=s_{T^{\prime \prime}}\left(x_{i}, L\left(T^{\prime \prime}\right)\right)>s_{T}\left(x_{i}, L(T)\right)=s_{T}(x, L(T))=l s(T)$, a contradiction. This shows that $a_{r}<\frac{|L(T)|}{2}$. 
Note that the branches of $T^{*}-y_{j_{0}}$ are $B_{k}$ with $1 \leq k \leq r-1$ and $k \neq j_{0}, T\left[\{x\} \cup V\left(B_{r}\right)\right]$. Then $b w_{T^{*}}\left(y_{j_{0}}\right)=$ $a_{r} \leq \frac{|L(T)|-1}{2}=\frac{\left|L\left(T^{*}\right)\right|}{2}$. So $y_{j_{0}}$ is an $L\left(T^{*}\right)$-centroid vertex by Lemma 2.2. As $a_{r} \geq 2$, we have $\operatorname{ls}(T)=$ $s_{T}(x, L(T))<s_{T}(x, L(T))+a_{r}-1=s_{T^{*}}\left(y_{j_{0}}, L\left(T^{*}\right)\right)=l s\left(T^{*}\right)$, a contradiction.

Now Claim 2 follows by combining Cases i and ii.

Claim 3. Any internal vertex in the path from $x$ to $x_{i}$ has degree two in $T$.

Suppose that this is not true. Assume that the path from $x$ to $x_{i}$ is $x \ldots w_{1} w w_{t} \ldots x_{i}$. Let $N_{T}(w)=$ $\left\{w_{1}, \ldots, w_{t}\right\}$. Let $\beta$ be the number of leaves of $T$ in the branches of $T$ at $w$ not containing $w_{1}$ and $w_{t}$. Then $\beta \geq t-2 \geq 1$. Let $T^{\prime}=T-\left\{w w_{i}: 2 \leq i \leq t-1\right\}-\left\{x y_{i}: 2 \leq i \leq r-1\right\}+\left\{w_{t} w_{i}: 2 \leq i \leq t-1\right\}+\left\{w_{t} y_{i}:\right.$ $2 \leq i \leq r-1\}$. By Claim 2, $a_{1}=\cdots=a_{r}$. So $b w_{T^{\prime}}\left(w_{t}\right)=\max \left\{a_{1}, \ldots, a_{r-1}, a_{r}-\beta\right\}=a_{1}=a_{r}$, and $w_{t}$ is an $L\left(T^{\prime}\right)$-centroid vertex by Lemma 2.2. Thus

$$
\begin{aligned}
l s\left(T^{\prime}\right)-l s(T) & =s_{T^{\prime}}\left(w_{t}, L\left(T^{\prime}\right)\right)-s_{T}(x, L(T)) \\
& =a_{1}\left(d_{T}(x, w)+1\right)-d_{T}(x, w) \beta-\left(d_{T}(x, w)+1\right)\left(a_{r}-\beta\right) \\
& =\beta \\
& >0
\end{aligned}
$$

implying that $l s\left(T^{\prime}\right)>l s(T)$, a contradiction. Thus Claim 3 follows.

Let $z_{1}, \ldots, z_{s}$ be all the neighbors of $x_{i}$ outside the path $P$, where $z_{1}$ lies on the path from $x_{i}$ to $x$ in $T$. Let $n_{1}\left(n_{2}\right.$, respectively) be the number of leaves in the branch of $T$ at $x_{i}$ containing $x_{i-1}\left(x_{i+1}\right.$, respectively). Assume that $n_{1} \geq n_{2}$.

Claim 4. $n_{1}=n_{2}$.

Suppose that this is not true. Then $n_{1}>n_{2}$. Let $T_{1}=T-\left\{x y_{j}: 2 \leq j \leq r-1\right\}-\left\{x_{i} z_{j}: 1 \leq j \leq s\right\}+\left\{x_{i+1} y_{j}\right.$ : $2 \leq j \leq r-1\}+\left\{x_{i+1} z_{j}: 1 \leq j \leq s\right\}$. By Lemma 2.2 and Claim 3 and noting that $n_{2}<n_{1}<a_{r}$, we have $b w_{T_{1}}\left(x_{i+1}\right)=\max \left\{a_{1}, \ldots, a_{r-1}, n_{1}\right\}=a_{1}=b w_{T}(x) \leq \frac{|L(T)|}{2}=\frac{\left|L\left(T_{1}\right)\right|}{2}$, and so $x_{i+1}$ is an $L\left(T_{1}\right)$-centroid vertex. Thus $l s\left(T_{1}\right)=s_{T_{1}}\left(x_{i+1}, L\left(T_{1}\right)\right)=s_{T}(x, L(T))+n_{1}-n_{2}>s_{T}\left(x_{i}, L(T)\right)=l s(T)$. This proves Claim 4.

Let $T_{2}=T-\left\{x y_{2}, x_{i} z_{1}\right\}+\left\{x_{i-1} z_{1}, x_{i+1} y_{2}\right\}$ if $\delta_{T}(x)=r=3$ and $T_{2}=T-\left\{x y_{1}, x y_{2}\right\}+\left\{x_{i-1} y_{1}, x_{i+1} y_{2}\right\}$ otherwise. Note that there are $n_{1}+a_{1}$ leaves of $T$ in the branch of $T_{2}$ at $x_{i}$ containing $x_{0}$ and $n_{2}+a_{2}$ leaves in the branch of $T_{2}$ at $x_{i}$ containing $x_{d}$. By Claim 4, we have $n_{1}=n_{2}$, so $n_{1}+a_{1}=n_{2}+a_{2}$. Thus $b w_{T_{2}}\left(x_{i}\right)=a_{1}+n_{1} \leq \frac{\left|L\left(T_{2}\right)\right|}{2}$, implying that $x_{i}$ is an $L\left(T_{2}\right)$-centroid vertex by Lemma 2.2 . It follows that $l s\left(T_{2}\right)=s_{T_{2}}\left(x_{i}, L\left(T_{2}\right)\right) \geq s_{T}(x, L(T))+2 a_{1}>s_{T}(x, L(T))=l s(T)$, a contradiction. This shows that $x$ lies on some diametric path. Assume that $x_{i}=x$, where $1 \leq i \leq d-1$. Denote by $a$ the number of leaves in the branch of $T$ at $x_{i}$ containing $x_{i-1}$.

Suppose that $T$ is not a caterpillar. Let $z$ be any leaf of $T$ outside $P$ with $d_{T}(z, P) \geq 2$.

Claim 5. $d_{T}(z, P)=d_{T}\left(z, x_{i}\right)$.

Suppose that this is not true. Then $d_{T}\left(z, x_{j}\right)=d_{T}(z, P)$ for some $j \neq i$. Assume that $j<i$. Choose $j$ such that $i-j$ is as small as possible.

We show that $\delta_{T}\left(x_{j+1}\right)=\cdots=\delta_{T}\left(x_{i-1}\right)=2$ if $j<i-1$. Suppose to the contrary that $j<i-1$ and $\delta_{T}\left(x_{k}\right) \geq 3$ for some $k$ with $j<k<i$. By moving the leaf edges at $x_{k}$ from $x_{k}$ to $x_{1}$, we get a tree $T^{\prime}$ for which $x$ is still an $L\left(T^{\prime}\right)$-centroid vertex by Lemma 2.2, so $l s\left(T^{\prime}\right)>l s(T)$, which is a contradiction. Thus we indeed have $\delta_{T}\left(x_{j+1}\right)=\cdots=\delta_{T}\left(x_{i-1}\right)=2$ if $j<i-1$.

Let $u$ be the unique neighbor vertex of $z$. Let $T^{\prime}$ the tree obtained from $T$ by moving the leaf edges at $u$ from $u$ to $x_{1}$. If $x_{i}$ is also an $L\left(T^{\prime}\right)$-centroid vertex, then, as $d_{T}\left(x_{1}, x_{i}\right) \geq d_{T}\left(u, x_{i}\right)$ and $u \in L\left(T^{\prime}\right)$, we have $l s\left(T^{\prime}\right)=s_{T^{\prime}}\left(x_{i}, L\left(T^{\prime}\right)\right)>s_{T}\left(x_{i}, L(T)\right)=l s(T)$, a contradiction. Thus $x_{i}$ is not an $L\left(T^{\prime}\right)$-centroid vertex. Note that the branches of $T^{\prime}$ at $x_{i}$ not containing $x_{j}$ are just the branches of $T$ at $x_{i}$ not containing $x_{j}$, and that $x_{i}$ is 
an $L(T)$-centroid vertex. By Lemma $2.2, a+1=b w_{T^{\prime}}(x)>\frac{\left|L\left(T^{\prime}\right)\right|}{2}$ and $a \leq \frac{|L(T)|}{2}$. So $\frac{|L(T)|+1}{2}=\frac{\left|L\left(T^{\prime}\right)\right|}{2}<a+1 \leq$ $\frac{|L(T)|}{2}+1$, i.e., $a=\frac{|L(T)|}{2}$. Then $b w_{T}\left(x_{j}, L(T)\right)=b w_{T^{\prime}}\left(x_{j}, L\left(T^{\prime}\right)\right)=|L(T)|-a=\frac{|L(T)|}{2}$, so $x_{j}$ is an $L(T)$-centroid vertex and also an $L\left(T^{\prime}\right)$-centroid vertex by Lemma 2.2. Thus $l s\left(T^{\prime}\right)=s_{T^{\prime}}\left(x_{j}, L\left(T^{\prime}\right)\right)>s_{T}\left(x_{j}, L(T)\right)=l s(T)$, also a contradiction. Now Claim 5 follows.

By Claim 5, if an internal vertex, say $x_{s}$, of $T$ on $P$ different from $x_{1}, x_{d-1}, x_{i}$ has degree more than two in $T$, then it is incident to $\delta_{T}\left(x_{s}\right)-2$ leaf edges. In this case, move the leaf edges at $x_{s}$ from $x_{s}$ to $x_{1}$ if $s<i$ and to $x_{d-1}$ if $s>i$ to form a tree $T^{\prime \prime}$. By Lemma $2.2, x_{i}$ is an $L\left(T^{\prime \prime}\right)$-centroid vertex, so $l s\left(T^{\prime \prime}\right)>l s(T)$, a contradiction. Thus each internal vertex of $T$ on $P$ different from $x_{1}, x_{d-1}, x_{i}$ has degree two in $T$.

Note that the number of leaves in the branch of $T$ at $x_{i}$ containing $x_{i-1}$ is $a$. Let $p=\delta_{T}(u)-1$. Let $k$ be the number of leaves in the branch of $T$ at $x_{i}$ containing $w$. Let $n_{0}$ be the maximum number of leaves in a branch of $T$ at $x_{i}$ containing neither $x_{i-1}$ nor $w$.

Suppose that $a+p \leq \frac{|L(T)|+1}{2}$. Let $T^{\prime}$ be the tree obtained from $T$ by moving the leaf edges at $u$ from $u$ to $x_{1}$. By Lemma 2.2, $b w_{T^{\prime}}\left(x_{i}\right)=\max \left\{a+p, k-p+1, n_{0}\right\} \leq \max \left\{a+p, \frac{|L(T)|}{2}\right\} \leq \frac{|L(T)|+1}{2}$, and so $x_{i}$ is an $L\left(T^{\prime}\right)$-centroid vertex. Then we have $l s\left(T^{\prime}\right)=s_{T^{\prime}}\left(x_{i}, L\left(T^{\prime}\right)\right)>s_{T}\left(x_{i}, L(T)\right)=l s(T)$, a contradiction.

Suppose that $a+p>\frac{|L(T)|+1}{2}$, i.e., $p>\frac{|L(T)|+1}{2}-a$. We form a tree $T^{\prime \prime}$ by moving $\left\lfloor\frac{|L(T)|+1}{2}\right\rfloor-a$ leaf edges at $u$ from $u$ to $x_{1}$ and the remaining leaf edges at $u$ from $u$ to $x_{d-1}$. Note that $b w_{T^{\prime \prime}}\left(x_{i}\right)=\left\lfloor\frac{|L(T)|+1}{2}\right\rfloor \leq \frac{\left|L\left(T^{\prime \prime}\right)\right|}{2}$. Thus $x_{i}$ is also an $L\left(T^{\prime \prime}\right)$-centroid vertex by Lemma 2.2. So $l s\left(T^{\prime \prime}\right)=s_{T^{\prime \prime}}\left(x_{i}, L\left(T^{\prime \prime}\right)\right)>s_{T}\left(x_{i}, L(T)\right)=l s(T)$, also a contradiction.

It follows that $T$ is a caterpillar, so $|L(T)|=n-d+1$.

Claim 6. Each vertex from $x_{2}, \ldots, x_{d-2}$ different from $x_{i}$ has degree two in $T$ if $d \geq 4$.

Suppose that this is not true with $d \geq 4$. Then $\delta_{T}\left(x_{j}\right)>2$ for some $j$ with $2 \leq j \leq d-2$ and $j \neq i$. Assume that $j>i$. Let $N$ be the set of neighbors of $x_{j}$ outside $P$ in $T$. Let $T^{\prime}=T-\left\{x_{j} z: z \in N\right\}+\left\{x_{d-1} z: z \in N\right\}$. As $T$ is a caterpillar, the diameter of $T^{\prime}$ is $d$. It is easy to see that $x_{i}$ is an $L\left(T^{\prime}\right)$-centroid vertex, so we have $l s\left(T^{\prime}\right)>l s(T)$, a contradiction. Thus Claim 6 follows.

By Claim 6, each vertex from $x_{2}, \ldots, x_{d-2}$ different from $x_{i}$ has degree two in $T$ if $d \geq 4$. If $i=1, d-1$, then by Lemma 2.2 , we have $T \cong T_{n,(n-d+1) / 2}$, and so $l s(T)=\frac{(n-d+1) d}{2}$ if $n-d$ is odd, and $T \cong T_{n ;(n-d) / 2,(n-d+2) / 2}$, and so $l s(T)=\frac{(n-d) d}{2}+1$ if $n-d$ is even.

Suppose that $i \neq 1, d-1$.

Case 1. $n-d$ is odd.

If $\delta_{T}\left(x_{i}\right) \geq 3$, then there are $\delta_{T}\left(x_{1}\right)-1$ leaves in the branch at $x_{i}$ of $T$ containing $x_{0}$ and $\delta_{T}\left(x_{d-1}\right)-1$ leaves in the branch at $x_{i}$ of $T$ containing $x_{d}$. Suppose without loss of generality, $\delta_{T}\left(x_{1}\right) \leq \delta_{T}\left(x_{d-1}\right)$. We form a tree $T^{\prime}$ by moving a leaf edge at $x_{i}$ from $x_{i}$ to $x_{1}$. Evidently, $l s\left(T^{\prime}\right)=s_{T^{\prime}}\left(x_{i}, L\left(T^{\prime}\right)\right)>s_{T}\left(x_{i}, L(T)\right)=l s(T)$, a contradiction. Thus $\delta_{T}\left(x_{i}\right)=2$. By Lemma 2.2, we have $T \cong T_{n,(n-d+1) / 2}$, and so $l s(T)=\frac{(n-d+1) d}{2}$.

Case 2. $n-d$ is even.

If $\delta_{T}\left(x_{i}\right) \geq 4$, then there are $\delta_{T}\left(x_{1}\right)-1$ leaves in the branch at $x_{i}$ of $T$ containing $x_{0}$ and $\delta_{T}\left(x_{d-1}\right)-1$ leaves in the branch at $x_{i}$ of $T$ containing $x_{d}$. Suppose without loss of generality, $\delta_{T}\left(x_{1}\right) \leq \delta_{T}\left(x_{d-1}\right)$. We form a tree $T^{\prime}$ by moving a leaf edge at $x_{i}$ from $x_{i}$ to $x_{1}$. Evidently, $l s\left(T^{\prime}\right)=s_{T^{\prime}}\left(x_{i}, L\left(T^{\prime}\right)\right)>s_{T}\left(x_{i}, L(T)\right)=l s(T)$, a contradiction. Thus $\delta_{T}\left(x_{i}\right) \leq 3$. By Lemma 2.2, we have $T \cong T_{n ;(n-d) / 2,(n-d+2) / 2}$ if $\delta_{T}\left(x_{i}\right)=2$ and $T$ is isomorphic to a tree obtained from $T_{n-1,(n-d) / 2}$ by adding a leaf edge at $x_{i}$ if $\delta_{T}\left(x_{i}\right)=3$. In either case, $l s(T)=\frac{(n-d) d}{2}+1$.

The result follows by combining the above two cases.

As an immediate consequence of the previous theorem, we have 
Corollary 3.3. Let $T$ be a tree of order $n \geq 6$. Then

$$
l s(T) \leq\left\lfloor\frac{(n+1)^{2}}{8}\right\rfloor
$$

with equality if and only if $T \cong T_{n,\lceil n / 4\rceil}$ if $n$ is even or $n \equiv 3(\bmod 4)$, and $T \cong T_{n,(n-1) / 4}, T_{n,(n+3) / 4}$ if $n \equiv 1(\bmod 4)$.

Proof. Let $d$ be the diameter of $T$. If $d=2$, then $l s(T)=n-1<\left\lfloor\frac{(n+1)^{2}}{8}\right\rfloor$. Suppose that $d \geq 3$. If $n-d$ is even, then by Theorem 3.2, we have

$$
l s(T) \leq \frac{(n-d) d}{2}+1 \leq \frac{n^{2}}{8}+1<\left\lfloor\frac{(n+1)^{2}}{8}\right\rfloor .
$$

Suppose that $n-d$ is odd. By Theorem 3.2, we have

$$
\begin{aligned}
l s(T) & \leq f(d):=\frac{(n-d+1) d}{2} \\
& \leq \begin{cases}f\left(\frac{n+1}{2}\right)=\frac{(n+1)^{2}}{8} & \text { if } d \text { and } \frac{n+1}{2} \text { are even } \\
f\left(\frac{n-1}{2}\right)=f\left(\frac{n+3}{2}\right)=\frac{n^{2}+2 n-3}{8} & \text { if } d \text { is even and } \frac{n+1}{2} \text { is odd } \\
f\left(\frac{n}{2}\right)=f\left(\frac{n}{2}+1\right)=\frac{n^{2}+2 n}{8} & \text { if } n \text { is even }\end{cases} \\
& =\left\lfloor\frac{(n+1)^{2}}{8}\right\rfloor
\end{aligned}
$$

with equalities if and only if $T \cong T_{n,(n-d+1) / 2}$ with $d=\frac{n+1}{2}$ if $\frac{n+1}{2}$ is even, $d=\frac{n-1}{2}, \frac{n+3}{2}$ if $\frac{n+1}{2}$ is odd, and $d=\frac{n}{2}, \frac{n}{2}+1$ if $n$ is even.

\section{Maximum Leaf Status}

Theorem 4.1. Let $T$ be a tree of order $n \geq 2$. Then

$$
L S(T) \geq n-1
$$

with equality if and only if $T \cong P_{n}$.

Proof. Let $x$ be a leaf peripherian vertex of $T$. Every vertex other than $x$ lies on some path from $x$ to a leaf (including the leaves themselves), so $s_{T}(x, L(T)) \geq n-1$ with equality if and only if every edge of $T$ lies on exactly one path from $x$ to a leaf, equivalently, $T \cong P_{n}$.

For integers $n$, $a$ with $1 \leq a \leq n-2$, let $P_{n, a}$ be the tree of order $n$ obtained by identifying the center of a star $S_{a+1}$ and a terminal vertex of a path $P_{n-a}$. In particular, $P_{n, 1}=P_{n}$ and $P_{n, n-2}=S_{n}$.

Theorem 4.2. Let $T$ be a tree of order $n \geq 4$. Then

$$
L S(T) \leq\left\lfloor\frac{n^{2}}{4}\right\rfloor
$$

with equality if and only if $T \cong P_{n, n / 2}$ for even $n$, and $T \cong P_{n,(n-1) / 2}, P_{n,(n+1) / 2}$ for odd $n$. 
Proof. If $n=4$, then $T \cong P_{4}$ or $S_{4}\left(=P_{4,2}\right)$ and $L S\left(P_{4}\right)=3<4=L S\left(P_{4,2}\right)$. If $n=5$, then $T \cong P_{5}, P_{5,2}$ or $S_{5}$ $\left(=P_{5,3}\right)$ and $L S\left(P_{5}\right)=4<6=L S\left(P_{5,2}\right)=L S\left(P_{5,3}\right)$. So the result holds if $n=4,5$.

Suppose that $n \geq 6$. Let $T$ be a tree of order $n$ that maximizes the maximum leaf status. Note that the maximum leaf status of $P_{n, n / 2}$ for even $n$, and $P_{n,(n-1) / 2}$ or $P_{n,(n+1) / 2}$ for odd $n$ is $\left\lfloor\frac{n^{2}}{4}\right\rfloor$. If $T \cong P_{n}$, then $L S(T)=n-1<\left\lfloor\frac{n^{2}}{4}\right\rfloor$. If $T \cong S_{n}$, then $L S(T)=2(n-2)<\left\lfloor\frac{n^{2}}{4}\right\rfloor$. So $T$ is neither a path nor a star.

Let $x$ be a leaf peripherian vertex of $T$. By Lemma $2.3, x \in L(T)$. Let $P:=x_{0} \ldots x_{r}$ be a longest path of $T$ starting from $x_{0}=x$. Then $3 \leq r \leq n-2$. Note that $\delta_{T}\left(x_{i}\right) \geq 3$ for some $i$ with $1 \leq i \leq r-1$. By the choice of $T, \delta_{T}\left(x_{i}\right)=2$ for $i=1, \ldots, r-2$, as, otherwise, by moving an edge outside $P$ from $x_{i}$ to $x_{r-1}$ we get a tree $T^{\prime}$, for which we have $L S\left(T^{\prime}\right) \geq s_{T^{\prime}}\left(x, L\left(T^{\prime}\right)\right)>s_{T}(x, L(T))=L S(T)$, which is a contradiction. As $P$ is a longest path from $x$, all neighbors of $x_{r-1}$ except $x_{r-2}$ are leaves. Let $a=\delta_{T}\left(x_{r-1}\right)-1$. Then $T \cong P_{n, a}$, and

$$
L S(T)=s_{T}(x, L(T))=a(n-a)
$$

which is maximized to $\left\lfloor\frac{n^{2}}{4}\right\rfloor$ if and only if $a=\frac{n}{2}$ if $n$ is even, and $a=\frac{n-1}{2}, \frac{n+1}{2}$ if $n$ is odd.

Theorem 4.3. Let $T$ be a tree of order $n$ with diameter $d$, where $2 \leq d \leq n-1$. Let $t=\left\lceil\frac{2(n-1-d)}{d}\right\rceil$ for even $d$ and $t=\left\lceil\frac{2(n-1-d)}{d-1}\right\rceil$ for odd $d$. Then

$$
L S(T) \geq n-1+\left\lceil\frac{d t}{2}\right\rceil
$$

with equality if and only if $T$ is a tree with a diametric path between two leaves $x$ and $y$ and exactly $t$ hanging paths at vertices of the diametric path such that $L S(T)=s_{T}(x, L(T))=s_{T}(y, L(T))$ for even dt and $L S(T)=$ $\max \left\{s_{T}(x, L(T)), s_{T}(y, L(T))\right\}$ and $\left|s_{T}(x, L(T))-s_{T}(y, L(T))\right|=1$ for odd $d t$.

Proof. Let $P:=v_{0} v_{1} \ldots v_{d}$ be a diametric path in $T$. Let $L^{*}(T)=L(T) \backslash\left\{v_{0}, v_{d}\right\}$. Since every vertex in $V(T) \backslash V(P)$ lies on some path from a vertex in $P$ to a vertex in $L^{*}(T)$, we have

$$
\sum_{w \in L^{*}(T)} d_{T}(w, P) \geq|E(T)|-d=n-1-d
$$

with equality if and only if every edge of $T$ outside $P$ lies on a unique shortest path connecting a leaf and a vertex of $P$, that is, each vertex outside $P$ has degree one or two in $T$. For convenience, write $t=\left\lceil\frac{2(n-1-d)}{d}\right\rceil$ for even $d$ and $t=\left\lceil\frac{2(n-1-d)}{d-1}\right\rceil$ for odd $d$. By a result of Qiao and Zhan [14], $|L(T)| \geq t+2$. So $\left|L^{*}(T)\right| \geq t$. Then

$$
\begin{aligned}
L S(T) & \geq \max \left\{s_{T}\left(v_{0}, L(T)\right), s_{T}\left(v_{d}, L(T)\right)\right\} \\
& \geq \frac{s_{T}\left(v_{0}, L(T)\right)+s_{T}\left(v_{d}, L(T)\right)}{2} \\
& =d+\sum_{w \in L^{*}(T)} \frac{d_{T}\left(v_{0}, w\right)+d_{T}\left(v_{d}, w\right)}{2} \\
& =d+\frac{\left|L^{*}(T)\right| d}{2}+\sum_{w \in L^{*}(T)} d_{T}(w, P) \\
& \geq d+\frac{d t}{2}+n-1-d \\
& =n-1+\frac{d t}{2} .
\end{aligned}
$$


So

$$
L S(T) \geq n-1+\left\lceil\frac{d t}{2}\right\rceil .
$$

Suppose that $L S(T)=n-1+\left\lceil\frac{d t}{2}\right\rceil$. By the proof of (4.1), each vertex outside $P$ has degree one or two in $T$. Note also that $\left|L^{*}(T)\right|=t$. Otherwise, $\left|L^{*}(T)\right| \geq t+1$. So, by (4.1),

$$
\begin{aligned}
n-1+\left\lceil\frac{d t}{2}\right\rceil=L S(T) & \geq d+\frac{\left|L^{*}(T)\right| d}{2}+n-1-d \\
& \geq n-1+\frac{d(t+1)}{2},
\end{aligned}
$$

a contradiction. So $T$ is a tree with a diametric path $P$ and exactly $t$ hanging paths at vertices of $P$.

If $d t$ is even, then the three inequalities in (4.1) must be equalities, so $L S(T)=s_{T}\left(v_{0}, L(T)\right)=s_{T}\left(v_{d}, L(T)\right)$.

Suppose next that $d t$ is odd. From (4.1), we have

$$
\max \left\{s_{T}\left(v_{0}, L(T)\right), s_{T}\left(v_{d}, L(T)\right)\right\}-\frac{s_{T}\left(v_{0}, L(T)\right)+s_{T}\left(v_{d}, L(T)\right)}{2} \leq \frac{1}{2},
$$

i.e., $\left|s_{T}\left(v_{0}, L(T)\right)-s_{T}\left(v_{d}, L(T)\right)\right|=0,1$. As $\left|L^{*}(T)\right|=t$, we have $\left|s_{T}\left(v_{0}, L(T)\right)-s_{T}\left(v_{d}, L(T)\right)\right|=1$ by $(4.1)$.

From (4.1), we also have $L S(T)=\max \left\{s_{T}\left(v_{0}, L(T)\right), s_{T}\left(v_{d}, L(T)\right)\right\}$, as otherwise,

$$
\begin{aligned}
n-1+\left\lceil\frac{d t}{2}\right\rceil & =L S(T) \\
& >\max \left\{s_{T}\left(v_{0}, L(T)\right), s_{T}\left(v_{d}, L(T)\right)\right\} \\
& =\frac{s_{T}\left(v_{0}, L(T)\right)+s_{T}\left(v_{d}, L(T)\right)+1}{2} \\
& =n-1+\left\lceil\frac{d t}{2}\right\rceil,
\end{aligned}
$$

a contradiction.

Conversely, if $T$ is a tree with a diametric path between two leaves $x$ and $y$ and exactly $t$ hanging paths at vertices of the diametric path such that $L S(T)=s_{T}(x, L(T))=s_{T}(y, L(T))$ for even $d t$ and $\mid s_{T}(x, L(T))-$ $s_{T}(y, L(T)) \mid=1$ and $L S(T)=\max \left\{s_{T}(x, L(T)), s_{T}(y, L(T))\right\}$ for odd $d t$, then

$$
\begin{aligned}
L S(T) & =\max \left\{s_{T}(x, L(T)), s_{T}(y, L(T))\right\} \\
& =\left\lceil\frac{s_{T}(x, L(T))+s_{T}(y, L(T))}{2}\right\rceil \\
& =n-1+\left\lceil\frac{d t}{2}\right\rceil,
\end{aligned}
$$

as desired.

To illustrate the above theorem, we give three trees $T_{1}, T_{2}, T_{3}$ on $n$ vertices of diameter $d$ to show that trees for which the lower bound in Theorem 4.3 is attained exist for all values of $n$ and $d$ for $2 \leq d \leq n-1$. Let $t$ be defined as in Theorem 4.3. Let $P:=x_{0} \ldots x_{d}$ be a path, where $x=x_{0}, x_{d}=y$. For even $d$, let $T_{1}$ be the tree obtained from $P$ by attaching $t-1$ hanging paths of length $\frac{d}{2}$ and one hanging path of length $n-d-1-\frac{d}{2}(t-1)$ at $x_{d / 2}$. For odd $d$ and even $t$, let $T_{2}$ be the tree obtained from $P$ by attaching $\frac{t}{2}$ hanging paths of length $\frac{d-1}{2}$ at $x_{(d-1) / 2}$ and $\frac{t}{2}-1$ hanging paths of length $\frac{d-1}{2}$ and one hanging path of length $n-\frac{(d-1) t}{2}-\frac{d+3}{2}$ at $x_{(d+1) / 2}$. For odd $d$ and odd $t$, let $T_{3}$ be the tree obtained from $P$ by attaching $\frac{t-1}{2}$ hanging paths of length $\frac{d-1}{2}$ at $x_{(d-1) / 2}$ and $\frac{t-1}{2}$ hanging paths of length $\frac{d-1}{2}$ and one hanging path of length $n-d-1-\frac{(d-1)(t-1)}{2}$ at $x_{(d+1) / 2}$.

We also give in Figure 1 the only trees that minimize the maximum leaf status among trees of order 15 with diameter 8 . 


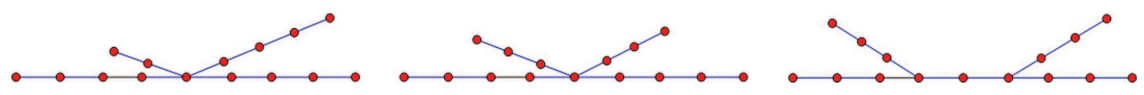

Figure 1. The order 15 diameter 8 trees with minimized maximum leaf status.

Theorem 4.4. Let $T$ be a tree of order $n$ with diameter $d$, where $2 \leq d \leq n-1$. Then

$$
L S(T) \leq d(n-d)
$$

with equality if and only if $T \cong P_{n, n-d}$.

Proof. The result is trivial if $d=2, n-1$. Suppose that $3 \leq d \leq n-2$. Note that $|L(T)| \leq n-d+1$ with equality if and only if $T$ is a caterpillar. Let $x$ be a leaf peripherian vertex of $T$. By Lemma $2.3, x \in L(T)$. Thus

$$
L S(T)=s_{T}(x, L(T))=\sum_{y \in L(T) \backslash\{x\}} d_{T}(x, y) \leq d \sum_{y \in L(T) \backslash\{x\}} 1 \leq d(n-d),
$$

with equalities if and only if $|L(T)|=n-d+1$ and the distance between $x$ and any other leaf is $d$, equivalently, $T$ is a caterpillar with a vertex, say $v$, of maximum degree $n-d+1$ such that $d_{T}(x, v)=d-1$, i.e., $T \cong P_{n, n-d}$.

\section{Minimum internal Status}

Proposition 5.1. Let $T$ be a tree of order $n \geq 3$.

(i) is $(T) \geq 0$ with equality if and only if $T \cong S_{n}$.

(ii) If $T ¥ S_{n}$, then is $(T) \geq 1$ with equality if and only if $T$ is a double star.

(iii) If $T ¥ S_{n}$, and $T$ is not a double star, then is $(T) \geq 2$ with equality if and only if $T$ is a caterpillar of diameter 4 .

Proof. Item (i) follows from the fact that $S_{n}$ is the only tree with exactly one internal vertex. Item (ii) follows as the double stars are the only trees with exactly two (adjacent) internal vertices. Item (iii) follows as any tree $T$ of diameter at least 4 contains three internal vertices inducing a path $P_{3}$ in $T$, and if $T$ has more than 3 internal vertices, then the subtree induced by internal vertices in $T$ contains $S_{4}$ or $P_{4}$ so that $i s(T) \geq 3$.

Furthermore, we have

Proposition 5.2. Suppose that $T$ is a tree of order $n$ with diameter $d$, where $2 \leq d \leq n-1$. Then

$$
i s(T) \geq\left\lfloor\frac{(d-1)^{2}}{4}\right\rfloor
$$

with equality if and only if $T$ is a caterpillar.

Proof. By Lemma 2.2, an $I(T)$-centroid vertex is an internal vertex of $T$.

Let $P:=v_{0} \ldots v_{d}$ be a diametric path in $T$. Obviously, $\left\{v_{1}, \ldots, v_{d-1}\right\}$ induces a path $P_{d-1}$ in $T$. If $T$ is a caterpillar, then $i s(T)=s\left(P_{d-1}\right)$. Suppose that $T$ is not a caterpillar, i.e., $I(T) \backslash V(P) \neq \emptyset$. For any $i=$ $1, \ldots, d-1$, we have $s_{T}\left(v_{i}, I(T)\right) \geq s\left(P_{d-1}\right)+\sum_{w \in I(T) \backslash V(P)} d_{T}\left(w, v_{i}\right)>s\left(P_{d-1}\right)$. Suppose that $w \in I(T) \backslash V(P)$. Assume that $d_{T}\left(w, v_{j}\right)=d_{T}(w, P)$ for some $2 \leq j \leq d-2$. Then we have

$$
s_{T}(w, I(T)) \geq s_{T}(w, I(T) \cap V(P))>s_{T}\left(v_{j}, I(T) \cap V(P)\right) \geq s\left(P_{d-1}\right) .
$$

Therefore, $i s(T) \geq s\left(P_{d-1}\right)=\left\lfloor\frac{(d-1)^{2}}{4}\right\rfloor$ with equality if and only if $T$ is a caterpillar. 
Theorem 5.3. Let $T$ be a tree of order $n \geq 3$. Then

$$
i s(T) \leq\left\lfloor\frac{(n-2)^{2}}{4}\right\rfloor
$$

with equality if and only if $T \cong P_{n}$.

Proof. By Lemma 2.2, an $I(T)$-centroid vertex is an internal vertex of $T$, so $i s(T)$ is equal to the minimum status of $T^{\prime}$, i.e., $i s(T)=s\left(T^{\prime}\right)$, where $T^{\prime}$ is the tree obtained from $T$ by deleting all leaves. Let $k=|L(T)|$. By Proposition 2.1 in [1], we have

$$
i s(T)=s\left(T^{\prime}\right) \leq\left\lfloor\frac{(n-k)^{2}}{4}\right\rfloor \leq\left\lfloor\frac{(n-2)^{2}}{4}\right\rfloor
$$

with equalities if and only if $k=2$ and $T \cong P_{n}$.

\section{Maximum internal Status}

Similar to Proposition 5.1, we have

Proposition 6.1. Let $T$ be a tree of order $n \geq 3$. The following statements are true.

(i) $I S(T) \geq 1$ with equality if and only if $T \cong S_{n}$.

(ii) If $T ¥ S_{n}$, then $I S(T) \geq 3$ with equality if and only if $T$ is a double star.

(iii) If $T ¥ S_{n}$, and $T$ is not a double star, then $I S(T) \geq 6$ with equality if and only if $T$ is a caterpillar of diameter 4 .

Furthermore, we have

Proposition 6.2. Suppose that $T$ is a tree of order $n$ with diameter $d$, where $2 \leq d \leq n-1$. Then $I S(T) \geq$ $\frac{d(d-1)}{2}$ with equality if and only if $T$ is a caterpillar.

Proof. Let $P:=v_{0} v_{1} \ldots v_{d}$ be a diametric path in $T$. Let $I^{*}(T)=I(T) \backslash\left\{v_{1}, \ldots, v_{d-1}\right\}$. Then

$$
\begin{aligned}
I S(T) & \geq \max \left\{s_{T}\left(v_{0}, I(T)\right), s_{T}\left(v_{d}, I(T)\right)\right\} \\
& \geq \frac{s_{T}\left(v_{0}, I(T)\right)+s_{T}\left(v_{d}, I(T)\right)}{2} \\
& =\frac{2 \sum_{i=1}^{d-1} i+\sum_{w \in I^{*}(T)}\left(d_{T}\left(v_{0}, w\right)+d_{T}\left(v_{d}, w\right)\right)}{2} \\
& \geq \frac{d(d-1)}{2}
\end{aligned}
$$

with equality if and only if $I^{*}(T)=\emptyset$, i.e., $T$ is a caterpillar.

Let $T$ be a tree with $u \in V(T)$. For positive integer $p$, we denote by $T_{u ; p}$ the tree consisting of $T$ and a path $P:=u u_{1} \ldots u_{p}$ such that $u$ is the only common vertex of $T$ and the path $P$. In this case, we also say that $P$ is a "hanging" path of length $p$ at $u$ in $T_{u ; p}$, though it is really a hanging path of length $p$ at $u$ in $T_{u ; p}$ only when $\delta_{T}(u) \geq 2$.

Let $G_{u ; 0}=G$. For nonnegative integer $p$ and $q$, let $G_{u ; p, q}=\left(G_{u ; p}\right)_{u ; q}$.

Lemma 6.3. Let $T$ be a nontrivial tree with $u \in V(T)$. Let $p$ and $q$ be positive integers with $p \geq q$. Then $I S\left(T_{u ; p+1, q-1}\right)=I S\left(T_{u ; p, q}\right)$ if $q \geq 2$ and $T$ is a star with center $u$, otherwise $I S\left(T_{u ; p+1, q-1}\right)>I S\left(T_{u ; p, q}\right)$. 
Proof. Let $H=T_{u ; p, q}$ and $H^{\prime}=T_{u ; p+1, q-1}$. Let $u_{0} u_{1} \ldots u_{p}$ and $v_{0} v_{1} \ldots v_{q}$ be the two "hanging" paths at $u$ in $H$, where $u_{0}=v_{0}=u$. Let $x$ be an internal peripherian vertex of $H$. Let $L^{*}(T)=L(T), I^{*}(T)=I(T)$ if $u \notin L(T)$ and $L^{*}(T)=L(T) \backslash\{u\}, I^{*}(T)=I(T) \cup\{u\}$ otherwise. Then $x \in L(H)=L^{*}(T) \cup\left\{u_{p}, v_{q}\right\}$ by Lemma 2.4. As $p \geq q$, we have $s_{H}\left(u_{p}, I(H)\right) \geq s_{H}\left(v_{q}, I(H)\right)$. So we may assume that $x \in L^{*}(T) \cup\left\{u_{p}\right\}$.

Case 1. $x \in L^{*}(T)$.

If $q \geq 2$, then

$$
\begin{aligned}
s_{H}(x, I(H)) & =\sum_{w \in I^{*}(T)} d_{H}(x, w)+\sum_{i=1}^{p-1}\left(d_{H}(x, u)+i\right)+\sum_{i=1}^{q-1}\left(d_{H}(x, u)+i\right) \\
& =\sum_{w \in I^{*}(T)} d_{H}(x, w)+d_{H}(x, u)(p+q-2)+\sum_{i=1}^{p-1} i+\sum_{i=1}^{q-1} i
\end{aligned}
$$

and

$$
\begin{aligned}
s_{H^{\prime}}\left(x, I\left(H^{\prime}\right)\right) & =\sum_{w \in I^{*}(T)} d_{H^{\prime}}(x, w)+\sum_{i=1}^{p}\left(d_{H^{\prime}}(x, u)+i\right)+\sum_{i=1}^{q-2}\left(d_{H^{\prime}}(x, u)+i\right) \\
& =\sum_{w \in I^{*}(T)} d_{H^{\prime}}(x, w)+d_{H^{\prime}}(x, u)(p+q-2)+\sum_{i=1}^{p} i+\sum_{i=1}^{q-2} i .
\end{aligned}
$$

Then $s_{H^{\prime}}\left(x, I\left(H^{\prime}\right)\right)-s_{H}(x, I(H))=p-q+1>0$ and thus $I S\left(H^{\prime}\right) \geq s_{H^{\prime}}\left(x, I\left(H^{\prime}\right)\right)>s_{H}(x, I(H))=I S(H)$. If $q=1$, then

$$
\begin{aligned}
s_{H}(x, I(H)) & =\sum_{w \in I^{*}(T)} d_{H}(x, w)+\sum_{i=1}^{p-1}\left(d_{H}(x, u)+i\right) \\
& =\sum_{w \in I^{*}(T)} d_{H}(x, w)+d_{H}(x, u)(p-1)+\sum_{i=1}^{p-1} i
\end{aligned}
$$

and

$$
\begin{aligned}
s_{H^{\prime}}\left(x, I\left(H^{\prime}\right)\right) & =\sum_{w \in I^{*}(T)} d_{H^{\prime}}(x, w)+\sum_{i=1}^{p}\left(d_{H^{\prime}}(x, u)+i\right) \\
& =\sum_{w \in I^{*}(T)} d_{H^{\prime}}(x, w)+d_{H^{\prime}}(x, u) p+\sum_{i=1}^{p} i .
\end{aligned}
$$

Then $s_{H^{\prime}}\left(x, I\left(H^{\prime}\right)\right)-s_{H}(x, I(H))=d_{H}(x, u)+p>0$ and thus $I S\left(H^{\prime}\right) \geq s_{H^{\prime}}\left(x, I\left(H^{\prime}\right)\right)>s_{H}(x, I(H))=$ $I S(H)$.

Case 2. $x=u_{p}$.

Let $q \geq 2$. Then

$$
\begin{aligned}
s_{H}(x, I(H)) & =\sum_{w \in I^{*}(T)}\left(p+d_{H}(u, w)\right)+\sum_{i=1}^{p-1} i+\sum_{i=1}^{q-1}(p+i) \\
& =\sum_{w \in I^{*}(T)}\left(p+d_{H}(u, w)\right)+p(q-1)+\sum_{i=1}^{p-1} i+\sum_{i=1}^{q-1} i
\end{aligned}
$$


and

$$
\begin{aligned}
s_{H^{\prime}}\left(x, I\left(H^{\prime}\right)\right) & =\sum_{w \in I^{*}(T)}\left(p+1+d_{H^{\prime}}(u, w)\right)+\sum_{i=1}^{p} i+\sum_{i=1}^{q-2}(p+1+i) \\
& =\sum_{w \in I^{*}(T)}\left(p+1+d_{H^{\prime}}(u, w)\right)+(p+1)(q-2)+\sum_{i=1}^{p} i+\sum_{i=1}^{q-2} i .
\end{aligned}
$$

Then $s_{H^{\prime}}\left(x, I\left(H^{\prime}\right)\right)-s_{H}(x, I(H))=\left|I^{*}(T)\right|-1$. If $\left|I^{*}(T)\right| \geq 2$, then $I S\left(H^{\prime}\right) \geq s_{H^{\prime}}\left(x, I\left(H^{\prime}\right)\right)>s_{H}(x, I(H))=$ $I S(H)$. If $\left|I^{*}(T)\right|=1$, then $I S\left(H^{\prime}\right)=s_{H^{\prime}}\left(x, I\left(H^{\prime}\right)\right)=s_{H}(x, I(H))=I S(H)$.

Let $q=1$. Then

$$
s_{H}(x, I(H))=\sum_{w \in I^{*}(T)}\left(p+d_{H}(u, w)\right)+\sum_{i=1}^{p-1} i,
$$

and

$$
s_{H^{\prime}}\left(x, I\left(H^{\prime}\right)\right)=\sum_{w \in I^{*}(T)}\left(p+1+d_{H^{\prime}}(u, w)\right)+\sum_{i=1}^{p} i .
$$

Then $s_{H^{\prime}}\left(x, I\left(H^{\prime}\right)\right)-s_{H}(x, I(H))=\left|I^{*}(T)\right|+p>0$, and so $I S\left(H^{\prime}\right) \geq s_{H^{\prime}}\left(x, I\left(H^{\prime}\right)\right)>s_{H}(x, I(H))=I S(H)$.

The result follows by combing the above two cases and noting that $\left|I^{*}(T)\right|=1$ if and only if $T$ is a star with center $u$.

Theorem 6.4. Let $T$ be a tree of order $n \geq 3$. Then

$$
I S(T) \leq \frac{n^{2}-3 n+2}{2}
$$

with equality if and only if $T \cong P_{n}$.

Proof. Let $T$ be a tree of order $n$ that maximizes the maximum internal status. Suppose that $T$ is not a path. Let $x \in L(T)$. Then we choose a vertex of degree at least three, say $u$, such that $d_{T}(x, u)$ is as large as possible. Then there are two hanging paths $P$ and $Q$ at $u$ in $T$. By Lemma 6.3, we can obtain a tree $T^{\prime}$ so that $I S\left(T^{\prime}\right)>I S(T)$, a contradiction. Thus $T \cong P_{n}$. Evidently, $I S\left(P_{n}\right)=\sum_{i=1}^{n-2} i=\frac{n^{2}-3 n+2}{2}$.

Theorem 6.5. Let $T$ be a tree of order $n$ with maximum degree $\Delta$, where $2 \leq \Delta \leq n-1$. Then $I S(T) \leq$ $\frac{1}{2}(n-\Delta)(n-\Delta+1)$ with equality if and only if $T$ is a starlike tree with at least $\Delta-2$ hanging paths of length one.

Proof. It is trivial if $\Delta=2$. Suppose that $\Delta \geq 3$. Let $T$ be a tree of order $n$ with maximum degree $\Delta$ that maximizes the maximum internal status. Let $u$ be a vertex of degree $\Delta$. If there is a vertex different from $u$ with degree at least three, then we may choose such a vertex $v$ by requiring that $d_{T}(u, v)$ is as large as possible. This implies that there are two hanging paths at $v$ in $T$. By Lemma 6.3, there is a tree of order $n$ with maximum degree $\Delta$ having larger maximum internal status, which is a contradiction. That is, $u$ is the only vertex of degree at least three. In other words, $T$ is a starlike tree. By Lemma 6.3 again, $\Delta-2$ hanging paths are of length one. Thus $T$ is a starlike tree with at least $\Delta-2$ hanging paths being of length one. The result follows by noting that $I S(T)=I S\left(P_{n, \Delta-1}\right)=\sum_{i=1}^{n-\Delta} i=\frac{1}{2}(n-\Delta)(n-\Delta+1)$.

Acknowledgements. We thank the referees for valuable comments and constructive suggestions. This work was supported by National Natural Science Foundation of China (No. 12071158). 


\section{REFERENCES}

[1] M. Aouchiche and P. Hansen, Proximity and remoteness in graphs: results and conjectures. Networks 58 (2011) 95-102.

[2] M. Aouchiche and P. Hansen, Proximity, remoteness and distance eigenvalues of a graph. Discrete Appl. Math. 213 (2016) $17-25$.

[3] M. Aouchiche and P. Hansen, Proximity, remoteness and girth in graphs. Discrete Appl. Math. 222 (2017) 31-39.

[4] F. Buckley and F. Harary, Distance in Graphs. Addison-Wesley Publishing Company, Redwood City, CA (1990).

[5] M. Cheng, H. Lin and B. Zhou, Minimum status of series-reduced trees with given parameters. Bull. Braz. Math. Soc. (N.S.) (2021) DOI: $10.1007 / \mathrm{s} 00574-021-00278-1$.

[6] A.A. Dobrynin and R. Sharafdini, Stepwise transmission irregular graphs. Appl. Math. Comput. 371 (2020) 124949.

[7] K. Durant and S. Wagner, On the centroid of increasing trees. Discrete Math. Theor. Comput. Sci. 21 (2019) Paper 8.

[8] A.N.C. Kang and D.A. Ault, Some properties of a centroid of a free tree. Information Process. Lett. 4 (1975) 18-20.

[9] C. Liang, B. Zhou and H. Guo, Minimum status, matching and domination of graphs. Comput. J. 64 (2021) $1384-1392$.

[10] H. Lin and B. Zhou, Which numbers are status differences? Appl. Math. Comput. 399 (2021) 126004.

[11] C. Lin, W.H. Tsai, J.L. Shang and Y.J. Zhang, Minimum statuses of connected graphs with fixed maximum degree and order. J. Comb. Optim. 24 (2012) 147-161.

[12] Z. Peng and B. Zhou, Minimun status of trees with given parameters. RAIRO: Oper. Res. 55 (2021) S765-S785.

[13] K. Pravas and A. Vijayakumar, The median problem on k-partite graphs. Discuss. Math. Graph Theory 35 (2015) $439-446$.

[14] P. Qiao and X. Zhan, The relation between the number of leaves of a tree and its diameter. Czechoslovak Math. J. (2021) DOI: $10.21136 /$ CMJ .2021.0492-20.

[15] R. Rissner and R.E. Burkard, Bounds on the radius and status of graphs. Networks 64 (2014) 76-83.

[16] J. Sedlar, Remoteness, proximity and few other distance invariants in graphs. Filomat 27 (2013) $1425-1435$.

[17] P.J. Slater, Centers to centroids in graphs. J. Graph Theory 2 (1978) 209-222.

[18] H. Smith, L. Székely, H. Wang and S. Yuan, On different "middle parts" of a tree. Electron. J. Combin. 25 (2018) Paper 3.17.

[19] D. Vukičević and G. Caporossi, Network descriptors based on betweenness centrality and transmission and their extremal values. Discrete Appl. Math. 161 (2013) 2678-2686.

[20] H. Wang, Centroid, leaf-centroid, and internal-centroid. Graphs Combin. 31 (2015) 783-793.

[21] B. Zelinka, Medians and peripherians of trees. Arch. Math. (Brno) 4 (1968) 87-95.

\section{Subscribe to Open (S20) A fair and sustainable open access model}

This journal is currently published in open access under a Subscribe-to-Open model (S2O). S2O is a transformative model that aims to move subscription journals to open access. Open access is the free, immediate, online availability of research articles combined with the rights to use these articles fully in the digital environment. We are thankful to our subscribers and sponsors for making it possible to publish this journal in open access, free of charge for authors.

\section{Please help to maintain this journal in open access!}

Check that your library subscribes to the journal, or make a personal donation to the S2O programme, by contacting subscribers@edpsciences.org

More information, including a list of sponsors and a financial transparency report, available at: https://www. edpsciences.org/en/maths-s2o-programme 